Revue d'histoire du XIXe siècle

Société d'histoire de la révolution de 1848 et des

révolutions du XIXe siècle

$52 \mid 2016$

Chrononymes. Dénommer le siècle

\title{
Introduction. Dénommer le siècle : « chrononymes » du XIX ${ }^{\mathrm{e}}$ siècle
}

\section{Dominique Kalifa}

\section{OpenEdition}

\section{Journals}

Édition électronique

URL : http://journals.openedition.org/rh19/4985

DOI : $10.4000 /$ rh 19.4985

ISSN : $1777-5329$

\section{Éditeur}

La Société de 1848

\section{Édition imprimée}

Date de publication : 1 juin 2016

Pagination : 9-17

ISSN : 1265-1354

Référence électronique

Dominique Kalifa «Introduction. Dénommer le siècle : «chrononymes » du XIXe siècle », Revue

d'histoire du XIXe siècle [En ligne], 52 | 2016, mis en ligne le 01 juin 2016, consulté le 22 septembre 2020. URL : http://journals.openedition.org/rh19/4985 ; DOl : https://doi.org/10.4000/rh19.4985 


\section{DOMINIQUE KALIFA}

\section{Introduction \\ Dénommer le siècle : "chrononymes" du XIX' siècle}

Le temps est la matière vive de l'histoire, mais les historiens n'en ont jamais eu le monopole. C'est même souvent d'ailleurs que sont venues les avancées majeures en la matière. Que l'on songe par exemple au rôle des anthropologues et des philosophes dans l'émergence des travaux sur la mémoire ${ }^{1}$. L'apport des linguistes, qui s'intéressent de longue date aux façons de dire et donc de penser le temps, s'avère tout aussi décisif. C'est notamment le cas des protocoles de dénomination, qui pèsent sur la perception des faits et participent à la construction du sens historique. Des travaux importants ont ainsi été consacrés aux "désignants évènementiels", ces éléments de discours qui servent à nommer et déterminent en conséquence des pans entiers de situations contextuelles. Mais ces termes, qui possèdent ce pouvoir singulier "d'éveiller la mémoire des faits par la seule mention du nom $»^{2}$, ne sont ni simples, ni neutres, et il importe donc d'en identifier la nature. Des concepts ont été proposés, qui permettent d'affiner la façon dont la langue appréhende et découpe la représentation du temps. Ainsi des «toponymes évènementiels", comme Tchernobyl, Outreau ou Auschwitz, qui circonscrivent sur un lieu donné ou emblématique toute une série d'événements qui le dépassent pourtant; ainsi des "héméronymes" ${ }^{3}$, qui font de même à partir d'une date (la Saint-Barthélemy, le 14 juillet, le 11 septembre); des "praxonymes» ${ }^{4}$, qui partent de la dénomination d'événements ou d'actions (la Guerre de Crimée, la Grande Dépression, l'Intifada); ou encore des "chrononymes", introduits en 1996 par la linguiste Eva Büchi pour désigner les "noms propres de temps" ${ }^{5}$. Ce dernier terme, mieux connu des historiens

1. Un seul exemple suffira, l'importance pour l'histoire des trois volumes de Temps et récit de Paul Ricœur (Paris, Seuil, 1983-1985).

2. Laura Calabrese, Le Rôle des désignants d'événements historico-médiatiques dans la construction de l'histoire immédiate. Une analyse du discours de la presse écrite, thèse de l'Université Libre de Bruxelles, 2010, p. 117.

3. Laura Calabrese, «Les héméronymes. Ces évènements qui font date, ces dates qui deviennent évènements", Mots. Les langages du politique, $\mathrm{n}^{\circ} 88,2008$, p. 115-128

4. Michelle Lecolle, Marie-Anne Paveau et Sandrine Reboul-Touré [dir.], Le nom propre en discours (Les carnets du Cediscor), Paris, Presses de la Sorbonne-Nouvelle, 2009.

5. Eva Büchi, Les Structures du "Französiches Etymologisches Wöterbuch". Recherches métalexicographiques et métalexicologiques, Tübingen, Niemeyer, 1996, p. 271. 
en raison de son rôle dans les opérations de périodisation, fut ensuite développé par Danièle Van de Velde et précisé en 2006 par Paul Bacot, Laurent Douzou et Marie-Anne Paveau. "Par chrononyme, expliquent-il dans un appel à communication ${ }^{7}$, nous entendons tout syntagme servant à désigner en propre une période de temps spécifique. Quelques exemples viennent rapidement à l'esprit : les Trente Glorieuses, l'Entre-deux-guerres, les Sixties, etc. " La parution deux ans plus tard d'une livraison de la revue Mots intitulée "Chrononymes. La politisation du temps" permit à la fois d'affiner la définition ("une expression, simple ou complexe, servant à désigner en propre une portion de temps que la communauté sociale appréhende, singularise, associe à des actes censés lui donner une cohérence, ce qui s'accompagne du besoin de la nommer $»^{8}$ ) et de la diffuser parmi les sciences sociales.

Evidemment, épistémologues et philosophes de l'histoire n'avaient pas attendu l'invention de ce terme pour comprendre que les divisions du temps, et la façon de les nommer, étaient des constructions qui n'avaient rien de naturel. Dès 1942, le britannique William H. Walsh avait qualifié de "colligatory concepts» (d'un terme qu'il emprunte d'ailleurs à l'historien des sciences victorien William Whewell) ces expressions qui regroupent des événements, des observations ou des données diverses sous une dénomination commune ${ }^{9}$. L'historien qui met en relation, assemble et nomme des éléments épars - Moyen Age, Renaissance, Première Guerre mondiale, etc. - façonne selon Walsh de tels concepts, qui servent à identifier des phénomènes originaux, à produire intelligibilité et explication. Walsh ne mettait qu'une condition à la création d'un "concept colligeant " : qu'il soit empiriquement vrai. Mais une telle exigence fut récusée par ses successeurs. Frank Ankersmit précisa à juste titre qu' un colligatory concept n'était ni vrai ni faux puisqu'il ne se réfèrait pas à des propos ou à des actes, mais à des constructions qui étaient de simples représentations historiques ${ }^{10}$. Herman Paul en a récemment donné une définition assez opératoire : « des concepts par lesquels les historiens mettent en ordre leurs récits du passé », «les noms propres de l'ordre que les historiens discernent dans une région ou une période spécifique ${ }^{11}$. La notion, on le voit, est assez proche de celle de

6. Danièle Van de Velde, «Existe-t-il des noms propres de temps?», Lexique, n 15, 2000, p. $35-45$.

7. http://calenda.org/197928

8. Paul Bacot, Laurent Douzou et Jean-Paul Honoré, "Chrononymes. La politisation du temps", Mots. Les langages du politique, $\mathrm{n}^{\circ}$ 87, 2008, p. 5-12.

9. William H. Walsh, 'The Intelligibility of History', Philosophy, volume 17, n 66, 1942, p. 128143. Walsh reprend et affine la notion dans 'Colligatory concepts in History', in William H. Burston and Donald Thomson (eds), Studies in the Nature of History, London, Routledge \& Kegan Paul, 1967, p. 65-84, repris dans Patrick L. Gardiner (ed.), The Philosophy of History, Oxford University Press, 1974, p. 127-144.

10. Frank Ankersmit, Narrative Logic. A Semantic Analysis of the Historian's Language, La Hague, Martinus Nijhoff, 1983.

11. "Concepts by which historians create order in their stories about the past", "the proper name 
chrononymes, même si l'on peut en discuter l'identité «conceptuelle»: des constructions d'historiens, conceptuellement élaborées, y côtoient en effet des expressions plus "vulgaires", venues du sens commun, du journalisme, de la politique ou de l'air du temps. «Moyen Age» ou "Belle Époque» ne me semblent pas de même nature que «Détente» ou «Restauration ». À cet égard, les termes proposés par les linguistes apparaissent plus précis et plus opératoires que cette notion.

Ces interrogations rejoignent également les très nombreuses réflexions menées par les historiens, depuis le milieu du XXe siècle, sur les questions de périodisation, de découpe et de scansions temporelles. Analysant «les destinées du mot "Moyen Age" ", Marc Bloch a consacré à ces aspects des pages lumineuses et célèbres d'Apologie pour l'histoire, dans lesquelles il insiste sur la dimension à la fois continuelle et changeante du temps, sur la vigueur des jugements qui vicient des classifications qui ne peuvent être autre chose que des étiquettes pédagogiques ou érudites. «Pas assez assurément, pour satisfaire, dans la recherche des divisions exactes, une science du réel $»^{12}$. Dans son sillage, François Furet invitait en 1971 à «revisiter les périodisations globales traditionnelles, qui sont pour l'essentiel un héritage idéologique du XIX ${ }^{e}$ siècle, et qui présupposent précisément ce qui reste à démontrer ${ }^{13}$. Des critiques vives, des propositions neuves, ont ainsi permis à l'historiographie de se débarrasser des carcans périodiques et de leurs pétrifications pour repenser à nouveaux frais les usages et les catégories du temps ${ }^{14}$. Des approches plus déliées en ont résulté, qui insistèrent sur les articulations, les chevauchements et la pluralité des temps ${ }^{15}$. L'histoire des concepts de Reinhart Koselleck (Begriffsgeschichte) fait ainsi de la sémantique historique un prérequis indispensable. Non pas seulement la saisie de la variabilité des mots, indicateurs privilégiés du changement social, ni les écarts entre usages actuels et usages passés, mais aussi les conditions et les enjeux sociaux, politiques, économiques dont ils sont le produit. À compter des années 1980, la réflexion s'est également étendue au champ de la mémoire et aux usages du passé, interrogeant, entre autres, le destin mémoriel des Camisards, de la Révolution française, de la Vendée ou de Vichy, puis à celui des "régimes d'historicité»" ${ }^{16}$. Séquences et temporali-

of an order that historians discern in a specific region or period", Herman Paul, Key Issues in Historical Theory, New York \& London, Routledge, 2015, p. 120

12. Marc Bloch, Apologie pour l'histoire ou métier d'historien, Paris, Colin, 1949, p. 102.

13. François Furet, "L'histoire quantitative et la construction des faits historiques ", Annales ESC, vol. $26, \mathrm{n}^{\circ} 1$, p. $74-75$.

14. Michel Foucault (L'Archéologie du savoir, Paris, Gallimard, 1969), Paul Veyne (L'Inventaire des différences, Paris, Seuil, 1976), François Furet (L'Atelier de l'histoire, Paris, Flammarion, 1982), Philippe Ariès (Le Temps de l'histoire, Paris, Ed. du Rocher, 1986) ont tonné contre les "périodisations totalitaires». Pour une approche synthétique de ces débats, $c f$. Krzysztof Pomian, L'Ordre du temps, Paris, Gallimard, 1984 et Jean Leduc, Les historiens et le temps, Paris, Seuil, 1999.

15. Reinhart Koselleck, Le Futur passé. Contribution à la sémantique des temps historiques (1979), Paris, Éd. EHESS, 1990

16. Philippe Joutard, La Légende des Camisards. Une sensibilité du passé, Paris, Gallimard, 1977; 
tés historiques ne sont donc pas, loin s'en faut, des points aveugles de la recherche historique ${ }^{17}$.

Il est cependant un secteur qui n'a pas suscité de réflexion d'ampleur, à tout le moins en France $^{18}$ : celui qui a trait aux noms et dénominations des séquences du contemporain, ainsi qu'aux imaginaires temporels qui s'y réfèrent. Alors que des travaux d'importance ont été consacrés aux usages et significations de catégories comme "Antiquité», "Moyen Age», "Renaissance", "Ancien Régime", la période contemporaine est restée à l'écart de ces interrogations. Or il ne s'agit pas d'une simple question d'érudition. Le risque d'anachronisme est ici redoutable tant la désignation d'une période, même réfléchie, charrie avec elle tout un imaginaire, une théâtralité, voire une "dramaturgie», qui peuvent en gauchir l'historicité propre et donc le sens. Elle peut aussi venir effacer des termes et des significations préexistants ou concurrents. Élucider ces chrononymes, leurs contextes et leurs modalités d'élaboration, leurs usages et leurs fonctions, apparait donc comme une indispensable opération historique. La chose est particulièrement importante pour les périodes postérieures à la Révolution française, marquées par la prolifération des discours ou des publications sur le temps et l'histoire. Le nombre de chrononymes, leurs usages et leur circulation ont donc fortement progressé. Or la plupart de ces termes continuent d'être utilisés de façon «naturelle». On connaît la formule célèbre de Lord Acton dans sa leçon inaugurale de juin 1895 : «Study problems, not periods», et la façon dont elle a, à juste titre, influencé l'historiographie. Mais il se trouve que dans le cas présent, la période est précisément le problème.

En s'attachant à sept des chrononymes les plus utilisés par l'historiographie du XIXe siècle - Vormärz, "Printemps des peuples», Risorgimento, Hungry Forties, Sexenio democratico, «fin-de-siècle» et «Belle Époque» - ce dossier voudrait contribuer à mieux éclairer l'histoire et le destin des noms du temps. L'objectif n'est pas de "dénoncer» l'artificialité de telles désignations, ni de "corriger» les représentations qu'elles charrient : une «belle époque», vraiment? des Hungry Forties, vraiment? De telles opérations sont évidemment nécessaires et il importe de savoir, à l'aune d'autres types de sources, que la «Belle Époque» fut une période de misère ouvrière et de tensions sociales, tout comme il importe de savoir que le miracle économique et consumériste

Jean-Clément Martin, La Vendée de la Mémoire, 1800-1980, Seuil, 1989; Henry Rousso, Le Syndrome de Vichy. De 1944 à nos jours, Paris, Seuil, 1990; Pierre Nora [dir.], Les Lieux de mémoires, Paris, Gallimard, 1984-1992; François Hartog, Régimes d'historicité. Présentisme et expériences du temps, Paris, Seuil, 2002.

17. Daniel Milo, Trahir le temps (Histoire), Paris, Les Belles Lettres, 1991 ; Patrick Boucheron, L'Entretemps. Conversations sur l'histoire, Lagrasse, Verdier, 2012.

18. La chose est différente en Angleterre, où la période victorienne a donné corps à un immense corpus de victoriana, que les historiens et les études culturelles ont pris à bras le corps. $C f$., parmi une production très abondante, John Kucich \& Diane Sadoff (eds), Victorian Afterlife. Postmodern Culture Rewrites the Nineteenth Century, Minneapolis, University of Minnesota Press, 2000; Christine L. Krueger, Functions of Victorian Culture at the Present Time, Ohio University Press, 2002, et surtout Cora Kaplan, Victoriana: Histories, Fictions, Criticism, New York, Columbia University Press, 2007. 
des «Trente Glorieuses» ne dura guère qu'une douzaine d'années ${ }^{19}$. Mais il s'agit là de démarches communément mises en œuvre et qui obéissent au cahier des charges habituel de l'historien. Plus neuf est en revanche de montrer que l'expression "Trente Glorieuses» scénarise une conception de la modernisation forgée par l'idéologie productiviste des technocrates et des aménageurs d'après-guerre ${ }^{20}$. L'objectif ne consiste pas davantage à «en finir avec...» de telles dénominations, ce qui serait à la fois présomptueux et illusoire. En dépit des raccourcis qu'ils produisent ou des imaginaires qu'ils charrient, ces termes sont aussi des faits d'histoire, produits par des sociétés qui les "chargent" de significations et d'enjeux de savoir. On n'en aura donc jamais «fini» avec eux. Il est essentiel en revanche d'expliciter les conditions (sociales, politiques, culturelles) de leur émergence, d'analyser leur usages, leurs traductions, leurs évolutions, de mettre au jour les éventuelles expressions concurrentes qu'ils ont pu évincer du discours historique. L'enquête n'est donc pas seulement historiographique ou lexicologique. Elle doit aussi porter sur les conflits, plus ou moins souterrains, que révèlent ces mises en mots, ainsi que sur leurs circulations sociales ou transnationales.

La lecture des articles qui suivent montre que tous les chrononymes ne se ressemblent pas. Une première distinction sépare ceux qui, comme "fin-desiècle» ou Risorgimento, sont contemporains des événements qu'ils relatent, de ceux, nettement plus nombreux (Vormärz, Hungry Forties, «Belle Époque») qui leur sont postérieurs et procèdent donc d'une lecture rétrospective et récapitulative, plus lourde d'appréciations anachroniques. Il convient également de différencier ceux qui sont le produit d'opérations historiographiques (ce qui les rapproche des colligatory concepts façon Walsh) comme Sexenio democratico, de ceux qui apparaissent comme des mots d'ordre politiques (Hungry Forties) ou des saisies de l'«air du temps» («Belle Époque»). Certains chrononymes affichent un jugement explicite sur la séquence qu'ils désignent («Belle Époque», Hungry Forties, Sexenio democratico), quand d'autres le font métaphoriquement («Printemps des peuples», Risorgimento) ou apposent un regard a priori plus neutre ("fin-de-siècle», Vormarz). Mais une fois attestés (il faudrait cependant élargir l'enquête pour suivre le destin des chrononymes qui n'ont pas "pris", des chrononymes ratés), tous sont rapidement essentialisés par le discours historique, des travaux les plus savants aux manuels, aux ouvrages pittoresques ou aux paratextes éditoriaux. Tous sont aussi l'objet d'usages, d'emplois et de réemplois pluriels et évolutifs. Ils sont donc clairement «historiques", c'est-à-dire passibles d'un questionnement historique, ce qui complique singulièrement la donne puisque, tout comme l'historiographie, les noms qui nous parlent du temps historique sont eux-mêmes pris

19. Rémy Pawin, "Retour sur les "Trente Glorieuses" et la périodisation du second XXe siècle», Revue d'histoire moderne et contemporaine, $\mathrm{n}^{\circ}$ 61, 2013, p. 155-175.

20. Ibidem, ainsi que Céline Pessis, Sezin Topçu, Christophe Bonneuil [dir.], Une autre histoire des «Trente Glorieuses». Modernisation, contestation et pollution dans la France d'après-guerre, Paris, La Découverte, 2013. 
dans le mouvement de l'histoire. Ce simple constat devrait convaincre de la nécessité de généraliser les enquêtes de ce type.

À première lecture, l'écart majeur semble être celui qui sépare les expressions endogènes, où événements, contextes et significations s'entremêlent, de toutes celles qui sont forgées après-coup. Historiquement, elles ne peuvent nous dire la même chose. Même si elles ne sont utilisées que par une étroite élite culturelle et sociale et n'ont qu'une diffusion très restreinte, les premières peuvent rendre compte d'une forme de conscience ou de "connaissance du temps $»^{21}$, partagée par des contemporains et portée par un "discours social ${ }^{22}$. Elles peuvent donc contribuer à nourrir une historicité propre, au sens que Marshall Sahlins donne à ce terme : "modalités de conscience de soi d'une communauté humaine ${ }^{23}$, ce qui n'est évidemment jamais le cas des expressions postérieures. Le Völkerfrühling dont Jean-Claude Caron montre la naissance dans la propagande unitaire et nationaliste allemande du premier XIX ${ }^{e}$ siècle ou la "fin-de-siècle» que traque Christophe Charle dans la France des années 1860-1880 peuvent aider à saisir des intentions et des contextes propres à leurs temps, à déchiffrer des fragments de conscience historique. Mais les choses se brouillent dès que le terme fait souche. Ses usages postérieurs rendent compte de reconstructions ou d'instrumentalisations souvent fort éloignées des significations initiales. C'est évidemment le cas du Risorgimento : si l'événement de référence semble évident (la création d'un État italien unifié), les contours et les facettes du processus n'ont jamais cessé de se réfracter depuis la proclamation du Royaume. Le cas du «Printemps des Peuples» est encore plus exemplaire : vidée de tout contenu social, l'expression est ensuite relue au seul prisme du national par Bismarck ou les pangermanistes, puis revivifiée tout autrement en 1948, dans le contexte de confiscation par le Kominform des mouvements politiques est-européens. En dirigeant cette année-là son 1848 en Europe, le printemps des peuples ${ }^{24}$, puis en contribuant vingt ans plus tard à populariser la notion de "printemps de Prague », l'historien hongrois François Fejtö, intellectuel antifasciste et antistalinien proche du "Congrès des intellectuels pour la liberté", invite à relire autrement les mouvements révolutionnaires du printemps 1848. La «fin-desiècle", par contraste, peut paraître moins instrumentalisée. Les significations ne s'en déplacent pas moins : initialement très pessimiste, l'expression se positive dans les années 1920. L'œuvre de Carl Schorske l'étend par la suite

21. C'est le nom de la première partie de l'Art Poétique de Paul Claudel (Paris, Mercure de France, 1907).

22. Au sens que lui a donné Marc Angenot dans 1889: un état du discours social, Longueil, Le Préambule, 1989, p. 13 : "Tout ce qui se dit et s'écrit dans un état de société; tout ce qui s’imprime, tout ce qui se parle publiquement [...] Ou plutôt, appelons "discours social" non pas ce tout empirique, cacophonique à la fois et redondant, mais les systèmes génériques, les répertoires topiques, les règles d'enchaînement d'énoncés qui, dans une société donnée, organisent le dicible - le narrable et l'opinable - et assurent la division du travail discursif".

23. François Hartog, Régimes d'historicité. Présentisme et expériences du temps, Paris, Seuil, 2003, p. 19.

24. François Fejtö [dir.], 1848 en Europe, le printemps des peuples, Paris, Minuit, 1948. 
à Vienne dans une acception assez distincte. Dans le monde anglophone, elle se substitue généralement à "Belle Époque», comme l'expliquent les auteurs d'un Fin-de-siècle Reader ${ }^{25}$. L'écrivain britannique Raymond Rudorff explique pour sa part, un peu vite sans doute, que les générations suivantes ont appelé «Belle Époque» ce que les contemporains appelaient «fin-desiècle ${ }^{26}$. Certains auteurs anglophones vont plus loin : à "Belle Époque», concept français nationaliste et impérialiste qui escamote la réalité, il faut opposer "fin de siècle» qui insiste au contraire sur une période chaotique faite d'anxiété, de répression, d'antagonisme sexuel et de tensions de classes ${ }^{27}$. Que le chrononyme soit initialement forgé par des contemporains ne suffit donc pas à l'exonérer des formes ultérieures d'instrumentalisation qui en altèrent la signification. Ce fut aussi le destin du Victorian age, tant dans la vision critique qu'en propose dès le début du XXe siècle le Bloomsbury group que dans les acceptions thatchériennes des années 1980, pour ne prendre que deux exemples d'une très riche vie posthume ${ }^{28}$. L'enquête devrait être étendue à d'autres de ces expressions d'époque, à l'image du Gilded age américain ${ }^{29}$.

Autant que les contextes d'émergence, il convient donc de traquer les fonctions et les usages d'expressions investies de significations politiques ou sociales spécifiques. Il peut s'agir de desseins assez transparents, comme dans le cas du Vormärz allemand, qui renvoie pour ses promoteurs à la révolution "manquée" de 1848 et à l'abandon des propositions d'unification qu'elle incarnait. L'échec du Vormärz et de son eschatologie politique ouvre la voie d'un Nachmärz porteur d'autres stratégies. Le temps est ici clairement politique, le vor-désignant la période qui précède les choix importants (les approches plus internes, plus culturelles ou plus continues de l'histoire des pays germaniques lui préferent l'expression Biedermeier). Il peut s'agir d'intentions simples, mais oubliées, comme celles qui s'attachent aux Hungry Forties. L'hypothèse initiale consistait à vérifier si un tel chrononyme inaugurait les décomptes décennaux du temps historique, dont on connaît l'importance au XXe siècle. Mais c'est une toute autre histoire qui surgit, celle d'un mot d'ordre politique lancé en 1903 par les militants free trade britanniques pour lutter contre les projets protectionnistes de Joseph Chamberlain. Forgé par la propre fille de Richard Cobden, il fabrique de la documentation historique (témoignages, lettres, souvenirs, etc.) dans une claire opération de

25. Mike Jay and Michael Neve (eds), 1900. A Fin-de-siècle Reader, London, Penguin Books, 1999. Mais on trouve aussi quelques acceptions françaises d'une «fin de siècle» durant jusqu'en 1914, par exemple Jean Roman, Paris fin de siècle, Paris, Robert Delpire, 1958.

26. Raymond Rudorff, Belle Époque. Paris in the Nineties, London, Hamilton, 1972, p. 13.

27. Jill Forbes and Michael Kelly, French Culturel Studies. An Introduction, Oxford University Press, 1995 , p. 37-38.

28. Cf. supra n. 17; Kelly Boyd and Rohan McWilliam, The Victorian Studies Reader, Abidgon, Routledge, 2007.

29. L'expresssion est forgée en 1873 par Mark Twain et Charles Warner (The Gilded Age: A Tale of Today). Cf. Bryant M. French, Mark Twain and the "Gilded age". The Book that Named an Era, Dallas, Southern Methodist University Press, 1965; Charles W. Calhoun (ed.), The Gilded Age. Perspectives on the Origins of Modern America, Lanham, Rowman and Littlefield, 2007. 
polémique politique : montrer que l'Angleterre mourait de faim avant l'abrogation des Corn laws. L'expression est ensuite naturalisée par la littérature, la politique et l'historiographie britanniques, jusqu'à apparaitre, en raison de sa forte capacité évocatrice et émotionnelle, comme un de ces "souvenirs" fabriqués, mais essentiels à l'identité anglaise.

La cas du Sexenio democratico espagnol (le moment de transition entre l'abdication de la reine Isabelle II en septembre 1869 et le retour des Bourbons en décembre 1874) est plus complexe. En apparence, c'est une construction d'historiens, un de ces colligatory concepts chargés d'introduire un peu d'ordre et d'intelligibilité analytique dans une séquence de grande confusion et d'expériences contradictoires. Mais sa naissance en 1973 et sa diffusion dans les années qui suivent la mort de Franco en 1975 la lestent d'une tout autre fonction : penser les transitions politiques, identifier dans le passé du pays l'existence de phases confuses mais stimulantes d'évolution démocratique. Quant à la «Belle Époque» qui émerge dans la France de l'Occupation et s'épanouit sous la IV éépublique, elle apparaît comme une figure de compensation capable de cristalliser dans l'épreuve une forme de bonheur "français", puis d'incarner une République triomphante à un moment où la puissance culturelle, internationale et coloniale était en péril. Elle prend par la suite d'autres visages, contestatrice, féministe, culturelle, patrimoniale, signalant à chaque fois la grande plasticité (et d'une certaine manière l'autonomie) d'un chrononyme capable de puiser à des sources différentes de l'imaginaire de référence.

Les «élucidations" que proposent les articles réunis dans ce dossier sont passionnantes et souvent très révélatrices des usages de l'histoire. On pourra sans doute s'interroger sur la finalité de l'entreprise : que nous permet-elle vraiment de mieux comprendre? De telles enquêtes me semblent ouvrir la voie à une compréhension plus exigeante et plus fine de l'histoire. Évoquons quelques pistes. Elles constituent d'abord un préalable, presque indispensable, à qui veut se dégager des projections anachroniques ou téléologiques insidieusement plaquées sur les temps passés. Toute histoire compréhensive devrait donc se défaire de la pesée des dénominations tout comme elle s'efforce d'ignorer ce qui est advenu par la suite ${ }^{30}$. Mais la démarche interroge à mon sens la nature même du travail historien, qui a finalement peu affaire au passé. Évanoui, mort, englouti, celui-ci est à jamais disparu et rien ne permettra jamais de le ressusciter. Le considérer comme un stock d'événements ou d'actions restituables est une illusion qui a parfois la vie dure, mais qui n'en reste pas moins une illusion. Des traces demeurent évidemment, et c'est la mission de l'histoire que de les expertiser. Mais travailler sur des traces s'inscrit toujours dans une perspective relationnelle, qui a le temps pour objet. Et celui-ci est rarement linéaire. Entre le présent de l'historien et

30. Alain Corbin, «Le risque, pour l'historien désireux de comprendre le passé, de savoir ce qui est advenu après la période qu'il étudie», Sociétés \& Représentations, n 40, 2015, p. 337-342. 
le passé de sa quête viennent s'immiscer une multitude d'autres temps, des présents et des passés d'hier qui interferent sur son objet. Décrypter les chrononymes, tout comme les autres formes de représentations de l'histoire, nous aide à considérer le passé pour ce qu'il est : une réalité mobile, changeante, «historique», travaillée par les hommes et les femmes qui l'ont habité, mais aussi par les regards, les lectures, les déplacements que les époques ultérieures lui ont fait subir. Ils nous invitent à penser notre rapport au passé dans un entrelacs d'interactions, nous aident à comprendre cet enchevêtrement de temporalités, cet «entretemps" presque kaléidoscopique qui est constitutif de l'Histoire. Ils nous invitent surtout à nous garder de tout triomphalisme : le regard ou l'expertise que nous portons aujourd'hui, aussi savant qu'il soit, ne constituera jamais qu'un moment, un fragment, au mieux une halte dans un processus relationnel qui ne retiendra bientôt de nous qu'une brève note infrapaginale.

Dominique Kalifa est professeur à l'Université Paris 1 Panthéon - Sorbonne (Centre d'histoire du XIX' siècle) et membre de l'Institut Universitaire de France 